\section{JTI}

JOURNAL OF

TRAUMA AND INJURY

\title{
Rib Fixation for a Patient with Severely Displaced and Overlapped Costal Cartilage Fractures
}

\author{
Sung Ho Han, M.D. ${ }^{1}$, Soon-Ho Chon, M.D., Ph.D. ${ }^{2}$, Jong Hyun Lee ${ }^{2}$, \\ Min Koo Lee, M.D. ${ }^{1}$, Oh Sang Kwon, M.D. ${ }^{1}$, Kyoung Hwan Kim, M.D. ${ }^{1}$, \\ Jung Suk Kim, M.D. ${ }^{1}$, Ho hyoung Lee, M.D. ${ }^{1}$ \\ Departments of ${ }^{1}$ Traumatology and ${ }^{2}$ Thoracic and Cardiovascular Surgery, Cheju Halla General \\ Hospital, Jeju, Korea
}

Received: September 28, 2017

Revised: January 4, 2018

Accepted: January 6, 2018

\section{Correspondence to}

Soon-Ho Chon, M.D., Ph.D.

Department of Thoracic and Cardiovascular Surgery, Cheju Halla General Hospital, 65 Doryeong-ro, Jeju 63127, Korea

Tel: +82-64-740-5039

Fax: +82-64-743-3110

E-mail:sh.chon@hotmail.com
Rib fixations for flail chest or displaced rib fractures are not a new technique. However, reports on rib fixations involving costal cartilage fractures are very few and surprisingly there are no reports of internal fixations involving only the costal cartilage in the English literature. The diagnosis is difficult and the necessity of the procedure may be quite controversial. Placing plates in screws into the costal cartilage alone may seem unstable and easily dislodged or stripped through the cartilage. We report a 31-yearold male scuba diver instructor who underwent rib fixations over his 7th and 8th costal cartilage ribs for severe pain. The procedure was done with conventional plates and screws. He had the plates and screws removed 2 months later due to lingering pain, but with them removed he is now quite happy with the results without pain. The procedure for fixation of painful overlapped costal cartilage is quite simple and can be done with the usual conventional methods, fixating plate and screws directly over the cartilage alone without fixation over the bony rib.

Keywords: Costal cartilage; Fracture dislocation; Rib fixation

Rib fixations for flail chest or displaced rib fractures are not a new technique. However, reports on rib fixations involving costal cartilage fractures are very few [1] and surprisingly there are no reports of internal fixations involving only the costal cartilage in the English literature. The diagnosis is difficult and the necessity of the procedure may be quite controversial. We report a 31-year-old male scuba diver instructor who (http://creativecommons.org/licenses/by-nc/4.0/) which permits unrestricted noncommercial use, distribution, and reproduction in any medium, provided the original work is properly cited.

\section{INTRODUCTION}




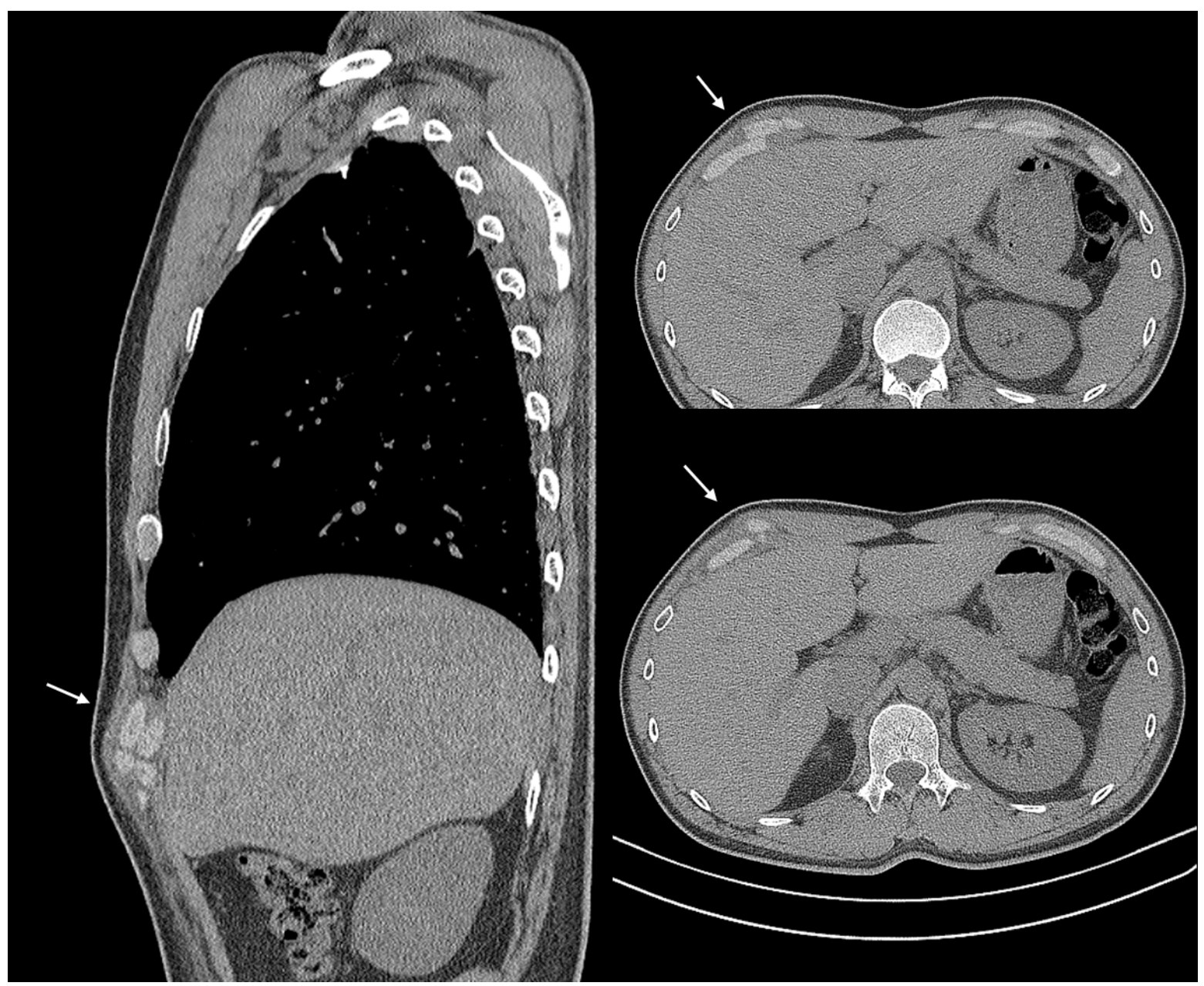

Fig. 1. Computer tomographic scan in the sagittal view shows the overlapping 7th and 8th costal ribs (arrows).

underwent rib fixations over his 7th and 8th costal cartilage ribs for severe pain. He had the plates and screws removed 2 months later due to lingering pain, but with them removed he is now quite happy with the results without pain.

\section{CASE REPORT}

A 31-year-old male patient had visited our hospital due to severe right parasternal chest wall pain due to blunt injury. He complained of extreme chest wall pain disproportionate to the findings seen radiographically. He was a scuba diver instructor and claimed that such pain would not allow him to continue with his occupation and his heart felt passion. His rib series could not reveal any fracture of his ribs. His computer tomographic scans presented overlapping and displacement of the 7 th and 8 th costal cartilages (Fig. 1). Both cartilage rib overlap was palpable and pain and tenderness was related to the areas over his overlapped 7th and 8th cartilage ribs. The patient had a Wong-Baker pain score of 8 despite routine intravenous nonsteroidal inflammatory drugs and intravenous narcotics. The health insurance provided by the Korean Government does not cover such an operation, thus, the patient had agreed to pay in full all the required fees related to the procedure.

The operation was done 11 days after admission and was straightforward. General anesthesia was done and exposure was done with a modest $8 \mathrm{~cm}$ incision between 


\section{JTI}

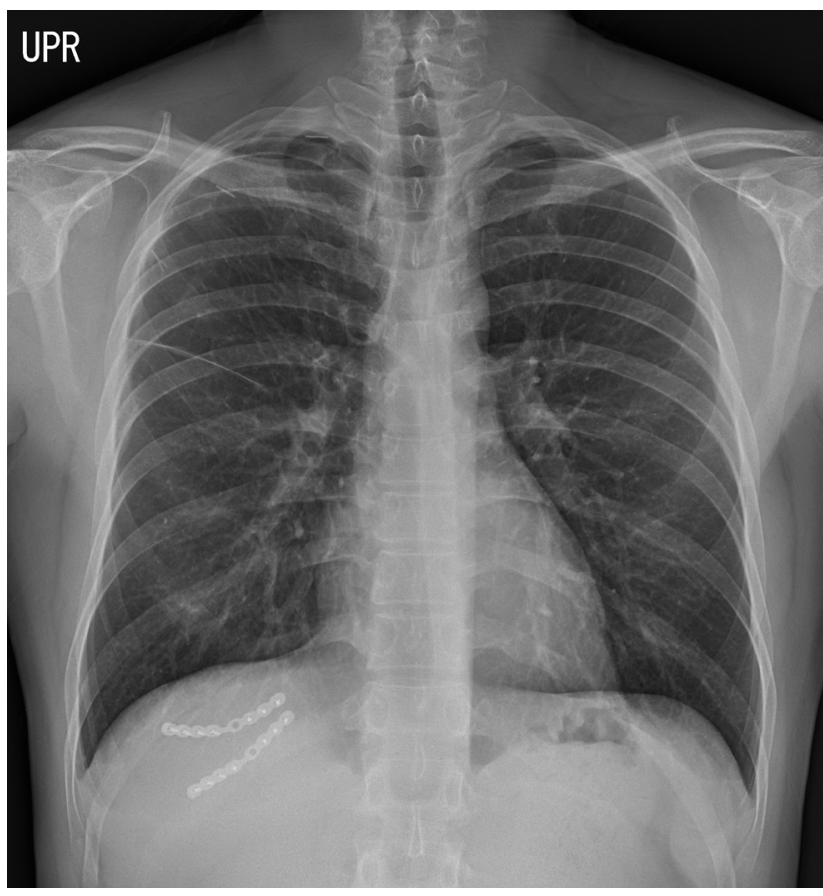

Fig. 2. The postoperative chest X-ray showing the fixated plate and screws in his 7th and 8th costal cartilages.

his 7th and 8th rib cartilage bed. The fractures were palpable and thus, finding the appropriate placement of the incision was not difficult. The cartilage portions were exposed and 8-hole titanium plate and 6 screws each were applied (SternaLock ${ }^{\circledR}$, Biomet, Jacksonville, FL, USA) just the same way it would be done over the costal bony ribs. The procedure took 56 minutes. A drain was placed and it was removed 3 days later. His postoperative $\mathrm{x}$-ray was unremarkable, showing the plate and screws (Fig. 2). At the request of the patient he was discharged one week after the operation. He was discharged with a Wong-Baker pain scale of 4 with narcotics per os.

Two months later at our outpatient clinic the patient still complained of pain related to the plate and screw internal fixations. Intraoperative findings presented complete healed cartilage fractures in both his 7th and 8th ribs. They were removed by general anesthesia without a drain and he was discharged two days after the procedure.

At outpatient follow up he was without pain and very happy with the results. There has been no need for follow up now two and a half years later.

\section{DISCUSSION}

Internal fixation of the ribs is not new and various methods have been reported. Patients with severe rib fractures and/or flail chest with internal fixation have shown promising results with decrease in hospital stays, intensive care unit stays, reduced incidence of pneumonia and septicemia, decreased chest deformity, reduced ventilator support and reduced mortality when compared to those who are treated conservatively [2].

The materials utilized for fixation has evolved from Judet struts to U-plates to plate and screws and intramedullary fixation devices such as the rib splint [3]. There is even a report of fixating ribs with absorbable plates [4]. There are reports of indications of rib fixation other than flail chest and these include three or more rib fractures with rib displacement of more than 1 rib cortical diameter, pulmonary function worsening with progressive volume loss on x-rays, mechanical ventilation, severe chest wall pain necessitating intravenous narcotics with visual analog score greater than 6 , lung impalement, open chest wall defect, need for thoracotomy, and pulmonary herniation [5]. The Denver Health Medical Center study described their indications for stabilization [6]. Their indications were 1) acute respiratory insufficiency despite optimal medical therapy, 2) uncontrolled pain despite optimal medical therapy, and 3) anticipated chronic pain/ impaired pulmonary mechanics. They have suggested fixation for greater than or equal to 3 severely displaced fractures of all ribs except for the first and second ribs and the floating 11th and 12th ribs. The operative indication in our particular case was a Wong-Baker pain scale of greater than 7 and two overlapping costal cartilage ribs greater than one diameter of the corresponding ribs. The patient was very sensitive to pain, especially during his scuba dives, which included foreign body sensation related pain. The pain would be difficult to ascertain whether it was due to fracture itself or due to the plate, however, considering his overlapped nonunion cartilage rib fractures completely healed and the fact that his pain subsided after the plate and screw removal, do suggest it was related to the plates and screws.

Our particular case presented displacement and overlapping of two costal cartilage ribs in his 7th and 8th rib 
with severe pain. The procedure was straightforward and done as would be done with costal bony ribs. There have been no reports seen on internal fixation of only the cartilaginous rib in the English literature and thus has inspired the authors in this case study.

\section{REFERENCES}

1. Bonne SL, Turnbull IR, Southard RE. Technique for repair of fractures and separations involving the cartilaginous portions of the anterior chest wall. Chest 2015;147:e199204.

2. Schulte K, Whitaker D, Attia R. In patients with acute flail chest does surgical rib fixation improve outcomes in terms of morbidity and mortality? Interact Cardiovasc Thorac
Surg 2016;23:314-9.

3. Fitzpatrick DC, Denard PJ, Phelan D, Long WB, Madey SM, Bottlang M. Operative stabilization of flail chest injuries: review of literature and fixation options. Eur J Trauma Emerg Surg 2010;36:427-33.

4. Nolasco-de la Rosa AL, Mosiñoz-Montes R, Matehuala-García J, Román-Guzmán E, Quero-Sandoval F, Reyes-Miranda AL. Unstable thorax fixation with bioabsorbable plates and screws. Presentation of some cases. Cir Cir 2015;83:23-8.

5. Chan EG, Stefancin E, Cunha JD. Rib fixation following trauma: a cardiothoracic surgeon's perspective. J Trauma Treat 2016;5:4-7.

6. Pieracci FM, Rodil M, Stovall RT, Johnson JL, Biffl WL, Mauffrey C, et al. Surgical stabilization of severe rib fractures. J Trauma Acute Care Surg 2015;78:883-7. 